\title{
Ka-Band Atmospheric Phase Stability Measurements in Goldstone, CA; White Sands, NM; and Guam
}

\author{
Michael J. Zemba, Jacquelynne R. Morse and James A. Nessel \\ NASA Glenn Research Center, Cleveland, OH, USA
}

\begin{abstract}
As spacecraft communication links are driven to higher frequencies (e.g. Ka-band) both by spectrum congestion and the appeal of higher data rates, the propagation phenomena at these frequencies must be well characterized for effective system design. In particular, the phase stability of a site at a given frequency will govern whether or not the site is a practical location for an antenna array, particularly if uplink capabilities are desired. Propagation studies to characterize such phenomena must be done on a site-by-site basis due to the wide variety of climates and weather conditions at each ground terminal. Accordingly, in order to statistically characterize the atmospheric effects on Ka-Band links, site test interferometers (STIs) have been deployed at three of NASA's operational sites to directly measure each site's tropospheric phase stability. Using three years of results from these experiments, this paper will statistically characterize the simultaneous atmospheric phase noise measurements recorded by the STIs deployed at the following ground station sites: the Goldstone Deep Space Communications Complex near Barstow, CA; the White Sands Ground Terminal near Las Cruces, NM; and the Guam Remote Ground Terminal on the island of Guam.
\end{abstract}

Index Terms - antenna arrays, radiowave propagation, propagation losses, interferometry, phase distortion.

\section{INTRODUCTION}

As electromagnetic waves propagate through the atmosphere, they experience amplitude and phase distortions due to atmospheric effects. Of particular interest to uplink array design is the atmospherically induced phase noise, which will manifest as a loss in the gain of the array due to loss of coherence between the individual elements of the array. For example, a two-element array ideally yields a two-fold or roughly $3 \mathrm{~dB}$ improvement over a single element, but phase distortions will reduce this relative to the level of distortion. Characterization of this reduction in directivity is one objective of atmospheric phase studies. Although compensation techniques have been developed for receive arrays, uplink compensation requires knowledge of current and future atmospheric conditions before transmission and is therefore more challenging to implement. Thus the phase stability of a site thus remains a primary concern when considering whether or not the site is a suitable location for an array with uplink capabilities [2].

Phase distortions occur predominantly in the troposphere, the lowest layer of Earth's atmosphere (up to $20 \mathrm{~km}$ above the surface depending on location) which contains the majority of Earth's weather phenomena [3]. There, inhomogeneous cells of water vapor are agitated by turbulent airflow, which introduces variation in refractivity along the path of

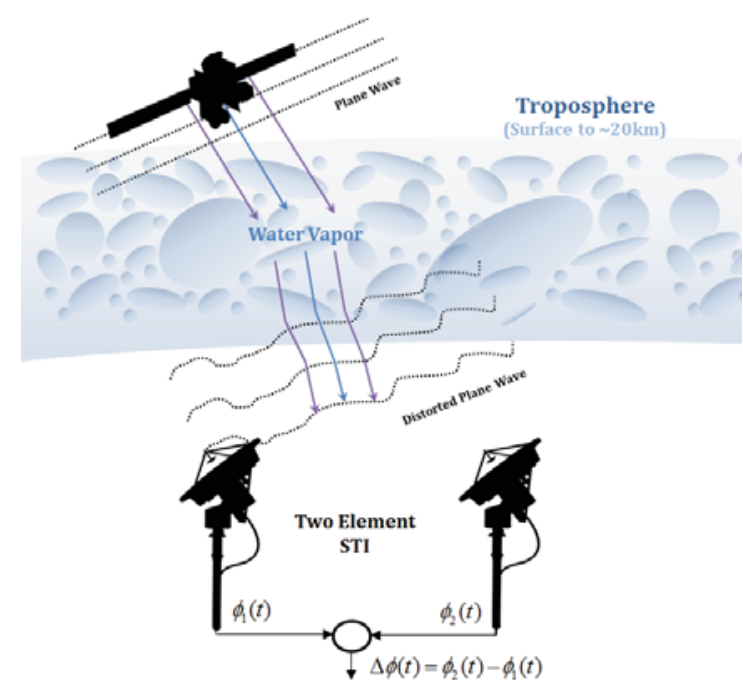

Fig. 1. Diagram of a site test interferometer experiment.

propagation as illustrated in the diagram of Fig. 1. This is the primary atmospheric mechanism affecting phase, distorting the wavefront and introducing phase differences between individual array elements. Like most meteorological phenomenon, these losses are stochastic and site-dependent [3].

This atmospheric phase instability can be measured through the use of a site test interferometer (STI). For this study, the STIs used consist of two spatially separated antennas observing the same unmodulated, continuous-wave (CW) beacon signal from a satellite in geostationary Earth orbit (GEO). The phase difference between the received signals is then used to derive the atmospheric phase effects, given that the signals originated from the same source and would be in phase if they had travelled equivalent path lengths [3]. NASA Glenn Research Center has deployed three such STIs to three different ground-station locations with the intent of characterizing their phase stability ahead of upgrades to KaBand operation [4]. These operational sites are located in Goldstone, California; White Sands, New Mexico; and on the island of Guam.

Using three years of data collected simultaneously from these campaigns, the goal of this study is to develop a characterization of the phase statistics of each site which may then be used as a metric of the sites' suitability for uplink arraying at Ka-Band. 
TABLE I. SITE PARAMETERS OF THE THREE STI LOCATIONS

\begin{tabular}{|l|c|c|c|}
\hline \multirow{2}{*}{ Installation Date } & May 2007 & Feb. 2009 & May 2010 \\
\cline { 2 - 4 } & Goldstone & White Sands & Guam \\
\hline Latitude & $35.2477^{\circ} \mathrm{N}$ & $32.5423^{\circ} \mathrm{N}$ & $13.5868^{\circ} \mathrm{N}$ \\
\hline Longitude & $116.7915^{\circ} \mathrm{W}$ & $106.6139^{\circ} \mathrm{W}$ & $144.8409^{\circ} \mathrm{E}$ \\
\hline Bearing & $90^{\circ}$ & $180^{\circ}$ & $170^{\circ}$ \\
\hline Baseline & $256 \mathrm{~m}$ & $208 \mathrm{~m}$ & $600 \mathrm{~m}$ \\
\hline Satellite & ANIK F2 & ANIK F2 & UFO-8 \\
\hline Elevation & $48.6^{\circ}$ & $51.8^{\circ}$ & $37.3^{\circ}$ \\
\hline Azimuth & $170.2^{\circ}$ & $188.3^{\circ}$ & $256.4^{\circ}$ \\
\hline Beacon Freq. & $20.2 \mathrm{GHz}$ & $20.2 \mathrm{GHz}$ & $20.7 \mathrm{GHz}$ \\
\hline
\end{tabular}

\section{INTERFEROMETER SPECIFICATIONS}

\section{A. Interferometer Design}

The design of the STIs, as implemented at each of the three sites, is presented in the block diagram of Fig. 2 from the front end to the analog-to-digital converter (ADC) [5]. Two antennas, a short baseline apart, receive the same unmodulated $\mathrm{CW}$ beacon signal from a GEO satellite. The first downconversion to $70 \mathrm{MHz}$ occurs directly at the feed after amplification, following which both signals are fed to a common intermediate frequency (IF) stage for further downconversion and processing. Two more stages down-convert the frequency to $455 \mathrm{kHz}$, after which they are sampled at a rate of 3.64 MHz with a $144 \mathrm{~ms}$ integration time. The output timeseries is then stored at 1 second intervals $(1 \mathrm{~Hz})$. The system was characterized in a laboratory environment without atmospheric contribution, and a root-mean-square (RMS) phase noise floor of $1.5^{\circ}$ or $0.21 \mathrm{ps}$ was observed [4].

\section{B. Site Specifications}

While the interferometer design is fundamentally the same at each location, the configurations of the STIs vary slightly from site-to-site for geographic as well as some logistic reasons. Specifications for the three sites and their associated satellites are presented in Table I. The primary differences between the three, with regards to phase measurement, are the elevation angle and baseline. Both have a notable impact on the phase measurement which must be normalized before comparisons between sites are made.

The STI at Goldstone was installed in May 2007 and sits on an east-west baseline of 256 meters. Here, as well as at the other sites, the baseline orientation is derived from the average direction of winds aloft [5]. The White Sands STI began operation in February 2009 and is located on a north-south baseline of 208 meters. These two STIs both observe the 20.2 $\mathrm{GHz}$ beacon on ANIK F2. The STI in Guam, deployed in May 2010, observes the $20.7 \mathrm{GHz}$ beacon on the UHF Follow-On 8 (UFO-8) satellite and sits on a north-south baseline of 600 meters. The significantly longer baseline in Guam is due to a

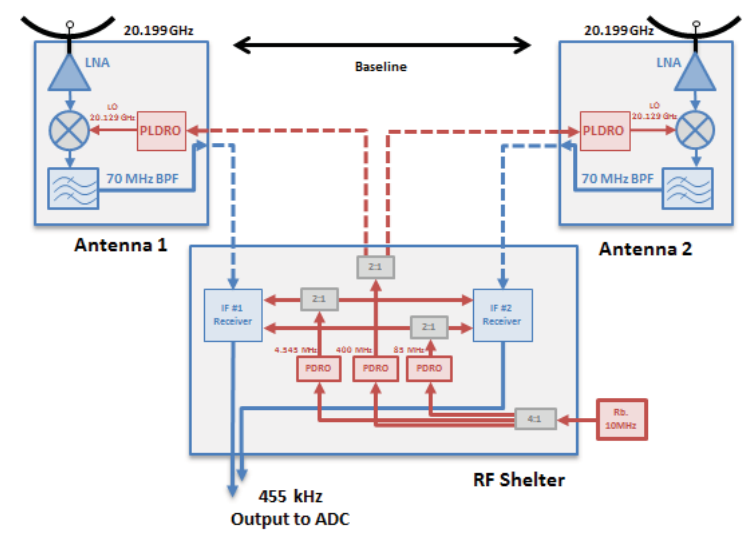

Fig. 2. Block diagram of the site test interferometer design.

concurrent site diversity study being conducted with the equipment [6]. The carrier-to-noise-density ratio (C/N0) at all locations was measured to be approximately $80 \mathrm{~dB}-\mathrm{Hz}$ [4].

\section{DATA PROCESSING}

\section{A. Phase Calibration}

Before analysis, certain undesired influences must be calibrated out of the phase data. The first step of calibration is to unwrap the phase data to remove its inherent $2 \pi$ wraps and obtain a continuous differential phase curve. Following this, a diurnal sinusoidal pattern dominates the continuous curve as seen in Fig. 3. This is introduced by slight motion of the satellite within the beam of the STI antennas. For this study, the motion of the observed satellites (ANIK F2 and UFO-8) is minor enough that it is contained within the beam of the antennas and no tracking is necessary; however, the resulting influence on the phase measurement must be removed in order to isolate the atmospheric contributions. Because the effects of satellite motion are slow-varying as compared to the atmospheric contributions, they may be removed (along with

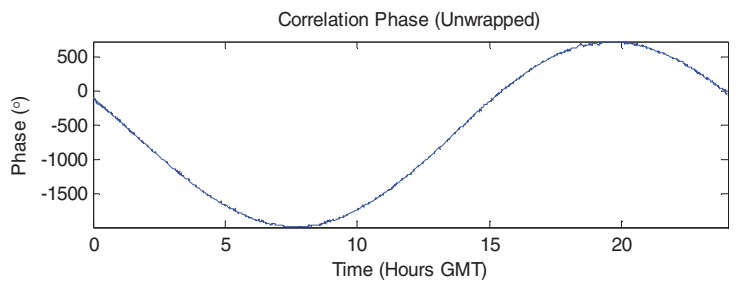

Phase with Trends Subtracted

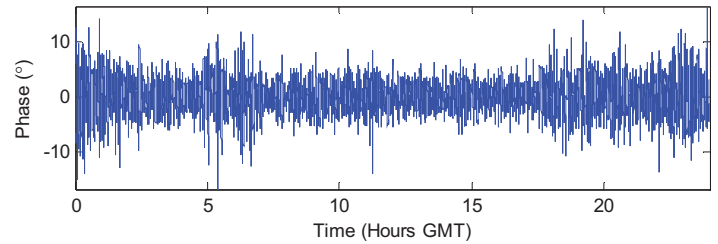

Fig. 3. 24 hours of phase measurements at White Sands before (above) and after (below) high-pass filtering. 
any other slow-varying influences, e.g. thermal drift) by means of high-pass filtering. This was implemented by subtracting $2^{\text {nd }}$ order polynomial fits from the data in 10-minute intervals [7].

\section{B. Normalization}

In order to compare the results from each site on equal footing, the data must be also normalized to account for variations such as baseline and elevation. The elevation angle of an STI is of concern when analyzing phase data because a signal received at a lower elevation angle will traverse a longer path through the atmosphere and therefore be subject to additional distortion. To normalize elevation angle, the phase data from each site was scaled to a reference angle of zenith $\left(90^{\circ}\right)$. This is implemented via the transformation:

$$
\sigma_{\phi}=\frac{\sigma_{\theta} \sin \theta}{\sin \phi}
$$

where $\sigma_{\square}$ is the phase at the measured elevation angle $\square$, and $\sigma_{\square}$ is the phase at the desired elevation angle of $\square$, and the thin layer Kolmogorov limit is assumed [8].

The baseline separation of the STI also presents a normalization concern as phase distortion will increase with separation distance. However, the baseline may be transformed through the use of the spatial structure function under the assumption of a frozen screen model [3]. The spatial structure function represents the variation of the phase with spatial separation and can be derived from the temporal structure function, which is calculated from the time-series measurements made by the interferometer. The transformation for baseline is given by:

$$
\sigma_{\phi}(r) \cong \sigma_{\phi}\left(r_{0}\right)\left(\frac{r}{r_{0}}\right)^{\alpha}
$$

where $r_{0}$ is the initial baseline, $r$ is the baseline to which the data will be transformed, and $\alpha$ is the Kolmogorov coefficient derived from the temporal structure function [2]. For this analysis, all baselines were normalized to a separation of $300 \mathrm{~m}$.

Although the variation in frequency is minor, the measurements were also normalized between the $20.2 \mathrm{GHz}$ beacons at Goldstone and White Sands and the $20.7 \mathrm{GHz}$ beacon in Guam. This can be accomplished by a simple ratio of the frequencies:

$$
\Delta \phi_{1}=\Delta \phi_{0} \frac{f_{1}}{f_{0}}
$$

where $f_{0}$ is the original frequency and $f_{1}$ is the frequency to which the measurement is scaled [9].

\section{Statistical Results}

The cumulative distribution function (CDF) of the normalized RMS phase at all three sites is presented in Fig. 4 for the three-year period of data collection. The $99^{\text {th }}$ percentile phase was $37.0^{\circ}$ for Goldstone, $38.5^{\circ}$ for White Sands, and $77.5^{\circ}$ for Guam. Alternatively, in terms of path length fluctuation, the variation was $1.53 \mathrm{~mm}$. $1.59 \mathrm{~mm}$. and 3.20 $\mathrm{mm}$, or in terms of time delay, $5.09 \mathrm{~ms}, 5.29 \mathrm{~ms}$, and $10.66 \mathrm{~ms}$, respectively.

With regards to seasonal analysis, Fig. 5 presents each site's $99^{\text {th }}$ percentile RMS phase plotted by month throughout the period of data collection. Goldstone and White Sands tend to have their highest phase instability during the summer, from June through October, while Guam has its best performance during this period.

In addition to seasonal variation, strong diurnal patterns were also observed, with the phase tending to be highest during the local morning and afternoon hours (8:00 - 16:00). Fig. 6 plots the average RMS phase at Goldstone, White Sands

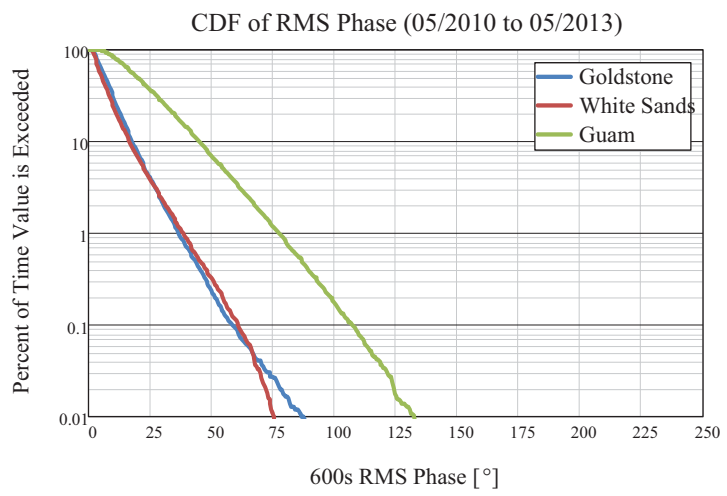

Fig. 4. Three-year RMS Phase CDFs.
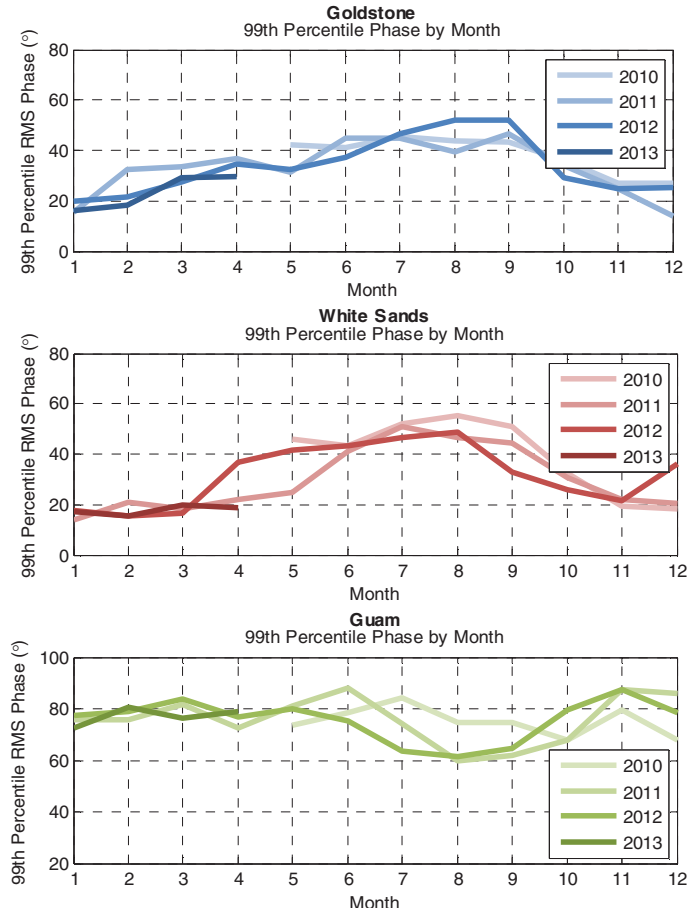

Fig. 5. $99^{\text {th }}$ Percentile RMS Phase Values by Month 
and Guam for each hour of the day at the $99^{\text {th }}, 95^{\text {th }}$, and $90^{\text {th }}$ percentile.

As a metric for comparison with these results, the Atacama Large Millimeter Array (ALMA) in Chile is located at one of the best sites in the world in terms of phase stability, due to the low humidity and high altitude of the Atacama Desert. In a similar interferometer study by Butler and Radford [10], the RMS phase near the ALMA site was found to be $1.05^{\circ}$ for the 10 th percentile, $1.91^{\circ}$ for the 25 th percentile, $3.92^{\circ}$ for the 50 th percentile, and $7.69^{\circ}$ for the 75 th percentile.

In terms of array loss, a two-element array of the same normalized configuration ( $300 \mathrm{~m}$ baseline, $90^{\circ}$ elevation angle) was simulated to derive the loss in gain that an array would sustain based on these phase statistics [2,9]. At Goldstone, the loss of the array was $0.2473 \mathrm{~dB}$ or better for $99 \%$ of the year, and likewise $0.2922 \mathrm{~dB}$ at White Sands and $0.6393 \mathrm{~dB}$ at Guam. By reducing the baselines of the array to $100 \mathrm{~m}$, the 99th percentile array loss was $0.0770 \mathrm{~dB}$ at Goldstone, 0.0864 $\mathrm{dB}$ at White Sands, and $0.1683 \mathrm{~dB}$ at Guam.

\section{CONCLUSIONS}

As indicated by these statistics, there is much similarity between the climatologically analogous Goldstone and White Sands sites. While Guam experiences much more phase instability as a tropical region, it is not necessarily prohibitive to uplink arraying. Also, although the phase noise is consistently higher, there is less variance throughout the year in Guam as compared to the two desert sites.

On a month-to-month basis, Goldstone and White Sands both experienced the lowest RMS phase during the winter months (November, December, January, February) and the highest during the summer months (June, July, August, September), as expected, with a standard deviation of $10.97^{\circ}$ throughout the year at Goldstone and $12.35^{\circ}$ at White Sands. Conversely, Guam was nearly the opposite with its lowest phase in summer months and its highest generally in the winter months, although the seasonal division was not as distinct. For Guam, the standard deviation of the phase RMS throughout the year was $8.80^{\circ}$.

\section{REFERENCES}

[1] K. McCarthy, F. Stocklin, B. Geldzahler, D. Friedman, P. Celeste, "NASA's Evolution to Ka-Band Space Communications for Near-Earth Spacecraft," SpaceOps 2010, Huntsville, AL, April 25 - 30, 2010.

[2] J. A. Nessel and R. J. Acosta, "Predicting Sparse Array Performance From Two-Element Interferometer Data," IEEE Trans. Antennas Propag., vol. 60, no. 2, pp. 886894, Feb. 2012.

[3] A. R. Thompson, J. M. Moran, and G. W. Swenson, Interferometry and Synthesis in Radio Astronomy. John Wiley \& Sons, 2001.

[4] R. J. Acosta, et al., "Simultaneous Ka-Band Site Characterization: Goldstone, CA, White Sands, NM, and
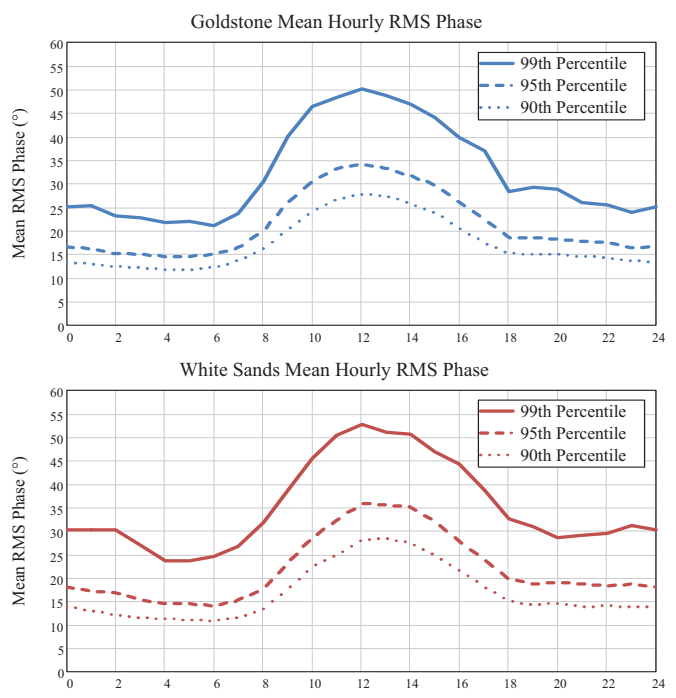

Guam Mean Hourly RMS Phase

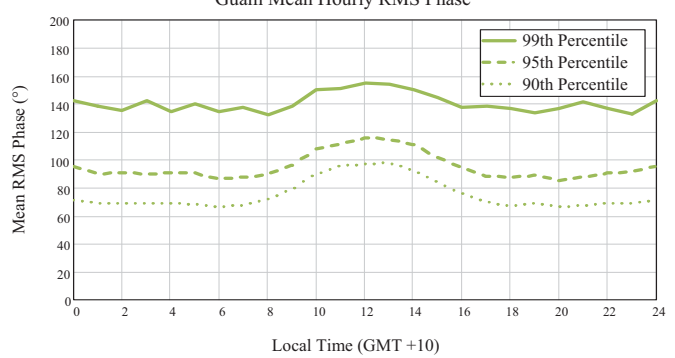

Fig. $6.99^{\text {th }}$ Percentile RMS Phase Values by Hour

Guam, USA," in 17th Ka and Broadband Communications Conference, Palermo, Italy, 2011.

[5] R. J. Acosta, B. D. Frantz, J. A. Nessel, and D. D. Morabito, "Goldstone Site Test Interferometer," in 13th $\mathrm{Ka}$ and Broadband Communications Conference, Turin, Italy, 2007.

[6] R. J. Acosta, J. R. Morse, M. J. Zemba, and J. A. Nessel, "Two Years of Site Diversity Measurements in Guam," in 18th $\mathrm{Ka}$ and Broadband Communications Conference, Ottawa, Canada, 2012.

[7] R. J. Acosta, J. A. Nessel, and D. D. Morabito, "Data Processing for Atmospheric Phase Interferometers," in 14th $\mathrm{Ka}$ and Broadband Communications Conference, Matera, Italy, 2008.

[8] Treuhaft, R. N. and Lanyi, G. E., "The effect of the dynamic wet-troposphere on radio interferometric measurements", Radio Science, Vol. 22, No. 2, pp. 251265, March-April, 1987.

[9] M. J. Zemba, "Site Characterization of Phase Instability via Interferometer Measurement," The University of Akron, Akron, $\mathrm{OH}$.

[10] B. J. Butler and S. J. E. Radford, "Atmospheric Phase Stability at Chajnantor and Pampa la Bola," NRAO ALMA Memo. No. 365, 2001. 